- Case Report

\title{
Benign Acute Childhood Myositis: Perplexing Complication after Acute Viral Pharyngitis
}

\author{
Sung-Won $\mathrm{Choi}^{1}{ }^{1 *}$, Hoon $\mathrm{Ko}^{2}$ \\ 'Department of Otorhinolaryngology and Biomedical Research Institute, Pusan National University Hospital, Busan, Korea \\ ${ }^{2}$ Department of Pediatrics and Biomedical Research Institute, Pusan National University Yangsan Hospital, Yangsan, Korea
}

Benign acute childhood myositis (BACM) is a rare transient condition that occurs in children during the early convalescent phase of a viral upper respiratory infection. BACM is self-limiting and characterized by sudden-onset bilateral calf pain that leads to difficulty in walking. We report a case of a 5-year-old boy with BACM who presented with acute-onset bilateral calf pain after a resolved episode of viral pharyngitis and subsequently refused to walk. With conservative treatment, the patient recovered completely after approximately 1 week. Although perplexing and challenging for clinicians unfamiliar with BACM, awareness of this rare clinical condition is essential to preventing unnecessary investigations and reassuring the patient and parents of its excellent prognosis.

Keywords: Myositis; Respiratory Tract Infections; Mobility Limitation; Differential Diagnosis

Received: September 19, 2017, Revised: October 10, 2017, Accepted: October 12, 2017

${ }^{*}$ Corresponding Author: Sung-Won Choi https://orcid.org/0000-0002-7463-7720

Tel: +82-51-240-7335, Fax: +82-51-246-8668, E-mail: gaelongs@gmail.com 


\section{INTRODUCTION}

Benign acute childhood myositis (BACM) is a rare self-limiting disease that primarily affects young school-aged boys and characteristically follows a viral upper respiratory infection (URI). It is also characterized by sudden-onset calf pain leading to a refusal to walk due to motor difficulty. ${ }^{1)}$ This refusal to walk can be perplexing for both clinicians and parents and commonly leads to an extensive work-up. Since many clinicians are unfamiliar with BACM, it is often misdiagnosed and interpreted as a more severe and complex disease. ${ }^{2,3)}$ The clinical manifestation is usually preceded by an initial phase of a viral URI, most commonly associated with influenza viruses. ${ }^{3)}$ The illness typically lasts for a brief period of time, usually up to 1 week. ${ }^{1)}$ Clinicians often treat many cases of patients with viral URI; however, BACM, a rare complication of viral URI, is still not well recognized. In this report, we present a case of BACM occurring in a 5-year-old boy in the early convalescence phase of a viral URI. We also review the literature regarding BACM and emphasize why knowledge of BACM is essential to preventing unnecessary invasive diagnostic procedures and therapeutic interventions, allowing clinicians to reassure patients and their parents about its excellent prognosis.

\section{CASE REPORT}

A previously healthy 5-year-old boy presented with a 1-day history of fever $\left(39^{\circ} \mathrm{C}\right)$, sore throat, anorexia, and malaise. The patient's immunizations were up to date. On examination, only a severe injected pharynx without exudates was observed. Initial laboratory investigations showed normal leukocytes $\left(6.38 \times 10^{9} / \mathrm{L}\right.$; normal range, 6 to $\left.15 \times 10^{9} / \mathrm{L}\right)$ and platelet counts $\left(257 \times 10^{9} / \mathrm{L}\right.$; normal range, 250 to $\left.350 \times 10^{9} / \mathrm{L}\right)$. However, the C-reactive protein level was elevated, confirming the presence of an infection ( $2.7 \mathrm{mg} / \mathrm{dL}$; normal range, 0 to $0.5 \mathrm{mg} / \mathrm{dL})$. A urinalysis showed normal results; no blood or proteins were detected. A nasopharyngeal aspirate (NPA) was taken to test for influenza A and $\mathrm{B}$; human parainfluenza virus types 1,2 , and 3 ; respiratory syncytial virus; adenovirus; and human metapneumovirus (routine panel) by polymerase chain reaction. The NPA results were positive for influenza A but no other viruses. Patients with flu do not ordinarily require hospital admission. However, the parent requested admission due to the fever and anorexia. On admission, the patient received symptomatic treatment with simple analgesics and intravenous fluids to prevent dehydration. Antiviral therapy was not considered because the patient had no risk factors such as asthma, cardiac disorders, or other conditions. During the 2-day hospitalization, the patient's symptoms resolved completely including the injected pharynx and his condition rapidly improved, allowing us to consider hospital discharge.

On the morning of the third day after admission, the patient was unable to get out of bed and unexpectedly refused to walk. The caregiver attempted to make the patient stand, but he was unable to bear weight on his legs and complained of bilateral calf pain. On examination, the patient was afebrile with normal vital signs and normal consciousness.
Furthermore, there was no evidence of trauma to the lower limbs. A neurological examination revealed normal muscle power and tone; the tendon reflexes and sensation in his lower limbs were also normal. However, the patient could not walk. A detailed examination failed to reveal any abnormalities other than calf tenderness, and no erythema or edema was noted. At rest, the patient kept his feet in slight plantar flexion since passive dorsiflexion at the ankles caused sharp pain. A laboratory investigation revealed a significantly increased creatine kinase (CK) level (2,690 U/L; normal range, 5 to $217 \mathrm{U} / \mathrm{L})$ and normal leukocyte count $\left(7.84 \times 10^{9} / \mathrm{L}\right.$; normal range, 6 to $\left.15 \times 10^{9} / \mathrm{L}\right)$ and C-reactive protein level $(0.3 \mathrm{mg} / \mathrm{dL}$; normal range, 0 to $0.5 \mathrm{mg} / \mathrm{dL})$. Complete blood count, electrolytes, blood urea nitrogen, creatinine, prothrombin time, partial thromboplastin time, and other routine blood parameters were also within normal limits. Urinalysis was normal with no detected myoglobin. After consulting the pediatric neurology department, we extended his admission for observation and conservative management. The patient was clinically diagnosed with BACM. Two days after its onset, the calf pain spontaneously resolved, and the patient was able to walk and run without support. CK levels gradually decreased, and a repeated urinalysis was negative for myoglobin. The patient was discharged 5 days after admission (2 days after BACM symptom onset). A repeat CK measurement 1 week later revealed normal levels, and the patient made a complete recovery with no residual impairments or neurological sequelae (Table 1).

\section{DISCUSSION}

The rare clinical entity BACM was first reported as "myalgia cruris epidemica" by Lundberg ${ }^{1)}$ in 1957 . He described 74 school-aged children with severe calf myalgia following a URI. During an influenza epidemic, BACM was distinct from the diffuse myalgias that are common during the course of influenza infection. ${ }^{1)}$ In most cases, BACM is self-limiting, with the myositis developing once the patient's viral URI is already resolving. ${ }^{1)}$ Although it most commonly occurs after an influenza $\mathrm{B}$ infection, BACM has also been associated with influenza A, parainfluenza, Coxsackie virus, herpes simplex virus, Epstein-Barr virus, and adenovirus infections. ${ }^{4)}$ Data from animal studies suggest that BACM is possibly related to direct invasion of the muscle tissue by the infecting virus and its subsequent inability to replicate within the myocytes. ${ }^{5)}$ The initial infection results in muscle fiber necrosis, which is sufficient to elevate CK levels; although no viruses could be detected in biopsy

Table 1. Laboratory findings from disease onset to follow-up

\begin{tabular}{lccc}
\hline & $\begin{array}{c}\text { Creatine kinase } \\
(5-217 \mathrm{U} / \mathrm{L})\end{array}$ & $\begin{array}{c}\text { C-reactive protein } \\
(0-0.5 \mathrm{mg} / \mathrm{dL})\end{array}$ & $\begin{array}{c}\text { White blood cell count } \\
\left(6-15 \times 10^{9} / \mathrm{L}\right)\end{array}$ \\
\hline Day 1 & - & 2.7 & 6.38 \\
Day 3 & 2,690 & 0.3 & 7.84 \\
Day 4 & 1,254 & 0.2 & 9.98 \\
Day 5 & 289 & 0.5 & 6.14 \\
Day 12 & 55 & 0.3 & 7.43
\end{tabular}

Normal ranges are given in parentheses. 
specimens, this suggests that the virus is unable to replicate in this tissue. ${ }^{5)}$ However, in some cases, influenza virus antigens were detected in muscle biopsies from patients, suggesting that these viruses can directly infect the muscle. ${ }^{6)}$ Thus, uncertainty remains as to whether the myositis is caused by direct viral action or immune-mediated mechanisms. ${ }^{7)}$

Most patients who develop BACM are children, although the condition has been described in adolescents. ${ }^{8)}$ Boys are affected more frequently than girls. ${ }^{7)}$ The clinical picture is usually preceded by influenza, with symptoms of URI such as fever, malaise, cough, sore throat, and rhinorrhea. ${ }^{2)}$ Patients present with sudden-onset intense bilateral calf pain; characteristically, tenderness and difficulty walking or a refusal to walk after a period of rest, such as upon awakening, occur. Serum CK level is consistently elevated in almost all patients and returns to normal within 1 month of symptom resolution. ${ }^{7)}$ All clinical symptoms spontaneously and rapidly resolve within a week (Table 2).

The differential diagnosis in pediatric populations for failure to weight-bear includes Guillain-Barré syndrome, rhabdomyolysis, dermatomyositis, polymyositis, and muscular dystrophy (Table 3). The lack of neurological deficits, urine myoglobin, and exanthema of the face, chest, or extensor surfaces of the extremities help differentiate BACM from other causes of muscle pain. ${ }^{9)}$ Despite the clinical course of BACM being well described, only $24 \%$ of participating clinicians were able to recognize BACM. ${ }^{3)}$ Our case was typical with respect to the clinical manifestations. However, clinicians may be confused by the appearance of such atypical symptoms after resolving viral URI since the general perception is that a viral URI can be treated without complications.

BACM does not require any medical therapy or invasive tests. When symptoms have resolved, the patient does not require any follow-up.

Table 2. Key features associated with benign acute childhood myositis ${ }^{1-7)}$

$$
\text { Key features }
$$

Sudden refusal to walk or gait-related abnormalities on awakening due to bilateral calf pain after resolving viral upper respiratory infection

Boys between 3 and 14 years of age are most commonly affected

Normal neurological exam (deep tendon reflex; motor and sensory examination)

Elevated serum creatine kinase level and normal urinalysis findings

Patient's feet in slight plantar flexion; passive dorsiflexion at the ankles elicits pain

With conservative management, all clinical symptoms are spontaneously and rapidly recovered within 1 week
Some authors recommend ordering rapid influenza tests, serum CK levels, and a urinalysis to assess for myoglobinuria. ${ }^{10)}$ These tests can verify BACM diagnosis as well as help exclude alternative diagnoses that may present similarly to BACM but would require different management. ${ }^{10)}$

The symptoms of BACM can be quite impressive and both perplexing and challenging for parents and clinicians. A prompt diagnosis of BACM made by evaluating the characteristic symptoms as well as the clinical history of the preceding viral URI is required to prevent unnecessary diagnostic procedures and reassure the patient and parents about its excellent prognosis. This case report will help increase awareness of BACM in patients after a resolving viral URI and may bring clinicians closer to understanding this rare complication of viral URI.

\section{CONFLICT OF INTEREST}

No potential conflict of interest relevant to this article was reported.

\section{ACKNOWLEDGMENTS}

The authors appreciate the cooperation of the patient and their parents, who provided their consent for this study. This work was supported by clinical research grant from Pusan National University Hospital 2018.

\section{REFERENCES}

1. Lundberg A. Myalgia cruris epidemica. Acta Paediatr 1957;46:18-31.

2. Koliou M, Hadjiloizou S, Ourani S, Demosthenous A, Hadjidemetriou A. A case of benign acute childhood myositis associated with influenza A (H1N1) virus infection. Clin Microbiol Infect 2010;16:193-5.

3. Rubin E, De la Rubia L, Pascual A, Dominguez J, Flores C. Benign acute myositis associated with H1N1 influenza A virus infection. Eur J Pediatr 2010;169:1159-61.

4. Rennie LM, Hallam NF, Beattie TF. Benign acute childhood myositis in an accident and emergency setting. Emerg Med J 2005;22:686-8.

5. Davis LE, Kornfeld M. Experimental influenza B viral myositis. J Neurol Sci 2001;187:61-7.

6. Farrell MK, Partin JC, Bove KE. Epidemic influenza myopathy in Cincinnati in 1977. J Pediatr 1980;96(3 Pt 2):545-51.

7. Mackay MT, Kornberg AJ, Shield LK, Dennett X. Benign acute childhood myositis: laboratory and clinical features. Neurology

Table 3. Differential diagnosis of benign acute childhood myositis ${ }^{1-7)}$

\begin{tabular}{|c|c|c|c|c|c|c|c|c|}
\hline Clinical disorder & Family history & $\begin{array}{l}\text { Onset after } \\
\text { febrile illness }\end{array}$ & Recent injury & $\begin{array}{l}\text { Absent tendon } \\
\text { reflex }\end{array}$ & Myoglobinuria & Cutaneous rash & $\begin{array}{c}\text { Muscle } \\
\text { weakness }\end{array}$ & $\begin{array}{l}\text { Normal creatine } \\
\text { kinase }\end{array}$ \\
\hline Benign acute childhood myositis & & 0 & & & & & & \\
\hline Guillain-Barré & & 0 & & 0 & & & 0 & 0 \\
\hline Rhabdomyolysis & & & 0 & & 0 & & & \\
\hline Dermatomyositis & & & & & & 0 & 0 & 0 \\
\hline Polymyositis & & 0 & & & & & 0 & 0 \\
\hline Muscular dystrophy & 0 & & & 0 & & & 0 & \\
\hline
\end{tabular}


1999;53:2127-31.

8. Garcia J. MRI in inflammatory myopathies. Skeletal Radiol 2000;29: 425-38.

9. Panghaal V, Ortiz-Romero S, Lovinsky S, Levin TL. Benign acute child- hood myositis: an unusual cause of calf pain. Pediatr Radiol 2008;38: 703-5.

10. Heiner JD, Ball VL. A child with benign acute childhood myositis after influenza. J Emerg Med 2010;39:316-9. 